\title{
Piégeage sexuel de la processionnaire du pin, Thaumetopoea pityocampa Schiff. (Lep., Notodontidae) par des attractifs de synthèse. Premiers essais dans le Sud-Ouest de la France
}

\author{
Jacques EINHORN, Pierre MENASSIER (*), Didier MICHELOT \& Jacques RIOM (*) \\ I.N.R.A. Laboratoire des Médiateurs chimiques, Domaine de Brouessy, Magny-les Hameaux, F 78470 \\ Saint-Rémy-lès-Chevreuse.
}

(*) I.N.R.A. Laboratoire de Sylviculture et d'Ecologie de la Pinede landaise, Domaine de l'Hermitage, CestasPierroton, F 33610 Gazinet.

Mots-clés additionnels : Phéromone synthétique, Acétoxy-1 hexadécen-13(Z) yne-11, Piégeage lumineux.

Sex trapping of the processionary moth Thaumetopoea pityocampa Schiff. (Lep., Notodontidae) with synthetic attractants. First experiments in the South-West of France.

(Z)-13-hexadecen-11-ynyl acetate has been recently identified as a potential sex pheromone of the processionary moth Thaumetopoea pityocampa Schiff. In the present work, the attractivity of this compound in the field has been demonstrated and compared to that of the E isomer. Effects of pheromone dose and trap location were also studied with a view to optimized trapping. First results are given on the comparison between sex and light trappint at various sites in South-West France.

Additional key-words: Sex attractant, Synthetic pheromone, (Z)-13-hexadecen-11ynyl acetate, Light trapping.

\section{INTRODUCTION}

La sylviculture du pin maritime, Pinus pinaster Ait., occupe une place importante dans l'économie du Sud-Ouest de la France et, en particulier, dans celle des Landes de Gascogne.

Parmi les insectes ennemis de $P$. pinaster, la chenille de la processionnaire du pin, Thaumetopoea pityocampa Schiff, peut être à l'origine de dégâts fortement préjudiciables. C'est pourquoi, en 1978, un vaste réseau de pièges lumineux a été mis en place dans le Sud-Ouest de la France pour la surveillance de ce ravageur. Depuis sa création, ce réseau est géré par le laboratoire de Sylviculture et d'Ecologie de la Pinède landaise de l'I.N.R.A. (Cestas-Pierroton).
Des travaux sur la biologie et le comportement des adultes de $T$. pityocampa (DEMOLIN, 1969) ont permis, par ailleurs, de prévoir l'utilisation du piégeage sexuel pour surveiller cet insecte. Dans cette perspective, l'identification récente - dans le cadre d'une collaboration francoespagnole (GUERRERo et al., 1981) - d'une phéromone sexuelle attractive pouvait être considérée comme une étape décisive.

Entreprise en 1981, la présente étude avait pour premier objectif de vérifier sur le terrain l'attractivité du principal composé identifié, l'acétoxy-1 hexadécen-13 (Z) yne-11 (1). Ce composé constituait le $1^{\text {er }}$ exemple de structure ényne découvert chez les phéromones d'insectes. Il était donc particulièrement intéressant d'en connaître l'activité réelle en 
piégeage, l'hypothèse d'un précurseur de structure plus classique (ène-allène) n'étant $a$ priori pas exclue. Il était, de plus, utile de comparer cette activité à celle de son isomère $E(2)$, qui risque d'être formé en faible proportion lors de la synthèse de $l$ (GiUERRERo et al., 1981 ; Michelot et al., 1982). Nous avons également pris en compte l'acétate de dodécanyle (3), dont la présence a été détectée par chromatographie en phase gazeuse - spectrométrie de masse dans certains extraits phéromonaux, - qui pourrait jouer un rôle dans l'attractivité sexuelle (fig.1).

1

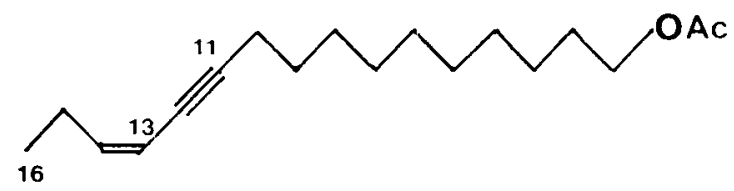

2

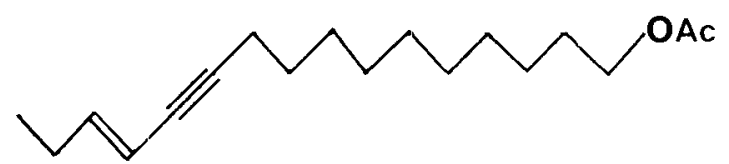

$\underline{3}$

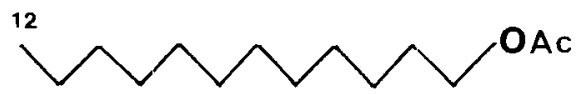

Figure 1

Composés étudiés.

Compounds studied.

En second lieu, notre étude comportait les 2 objectifs suivants :

- d'une part, effectuer une approche des conditions optimales pour le piégeage à l'aide de phéromone (doses d'attractif, hauteur des pièges, situation en lisière ou à l'intérieur du peuplement forestier).

- d'autre part, établir en plusieurs sites du massif landais, une $1^{\text {re }}$ comparaison entre les données obtenues au piège sexuel et celles fournies par le piégeage lumineux dans le cadre du réseau de surveillance de $T$. pityocampa. En effet, par rapport au piégeage lumineux, la mise au point du piégeage sexuel offrirait au moins 2 avantages importants: la grande sélectivité des captures et l'élimination de la contrainte qu'impose, au réseau de surveillance actuel, la fourniture d'énergie électrique. I! importe donc de définir les conditions dans lesquelles le piégeage sexuel peut être substitué au piégeage lumineux pour la détermination des dates optimales de traitement et des seuils de risque (corrélation entre captures et dégâts).

\section{MATÉRIEL ET MÉTHODES}

Les comparaisons de formulations ainsi que les études d'optimisation des conditions de piégeage (hauteurs, doses...) ont été réalisées à Cestas-Pierroton, sur le domaine de l'I.N.R.A. et sur des parcelles privées. Les comparaisons entre pièges lumineux et sexuels ont été faites sur des sites répartis dans tout le Sud-Ouest de la France (postes $5,8,10,14,18$ et 22 du réseau " piégeage lumineux », fig. 2).

Les pièges sexuels utilisés sont des pièges I.N.R.A. (STOCKEL, 1976; CUEVAS et al., 1981) de type « delta », avec volets latéraux ouverts, qui présentent une surface engluée de $500 \mathrm{~cm}^{2}$ (base interne). Ils sont appâtés avec des capsules

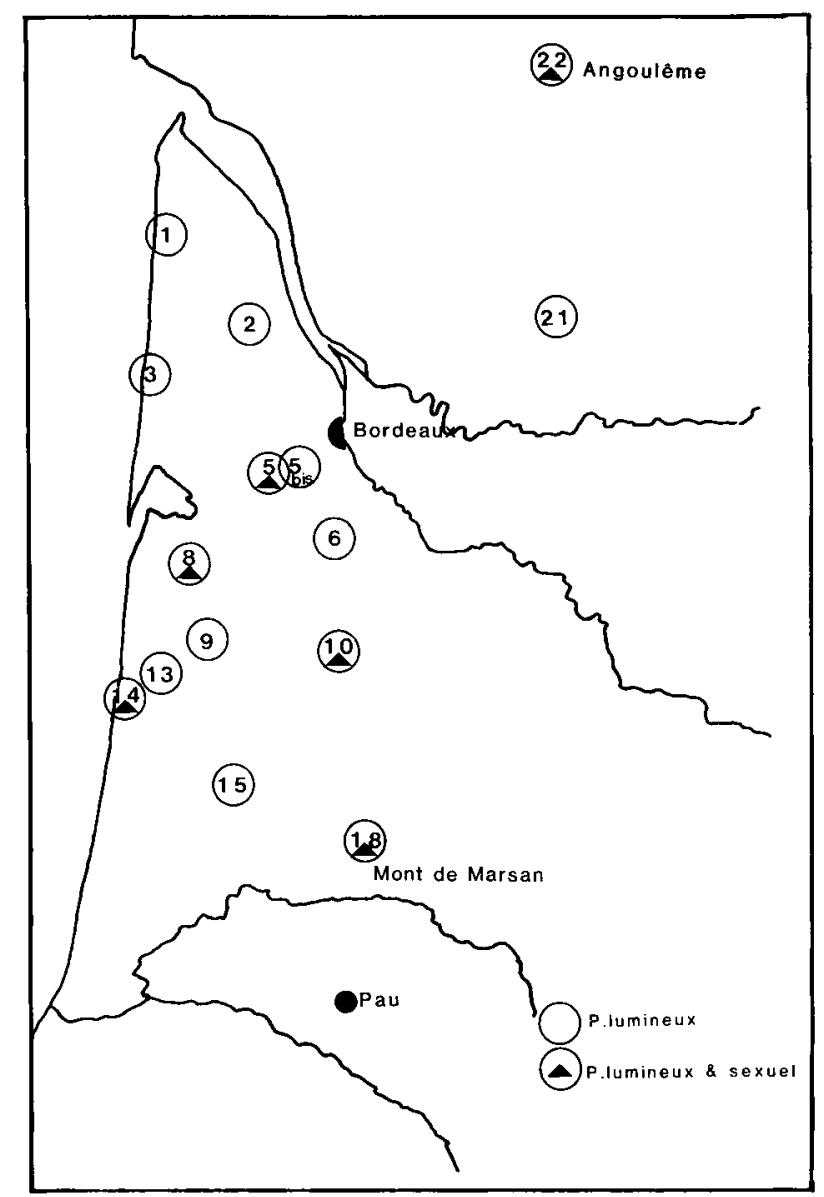

Figure 2

Nature et répartition géographique du réseau de surveillance de la processionnaire du pin en 1981 (Sud-Ouest).

Monitoring equipment for the Processionary Moth in 1981 (S.-W. France).

en caoutchouc (Ets LEUNE, Paris) préalablement lavées au méthanol et chargées à la dose de 1, 1,5 ou $2 \mathrm{mg}$ (composé 1) ou à une dose voisine de $1 \mathrm{mg}$ (mélanges de composés). L'acétoxy-1-hexadécen-13 (Z) yne-11 a été préparé d'après 2 voies de synthèse, conduisant à des degrés de pureté de 96 p. 100 (4 p. 100 d'isomère E, MiCHELOT et al., 1982) ou de 99,4 p. 100 (0,6 p. 100 d'isomère E, CAMPs et al., 1981). L'isomère $\mathrm{E}$ a été synthétisé avec un degré de pureté de 96 p. 100 (4 p. 100 d'isomère Z) (CAMPS et al., 1981). L'acétate de dodécanyle a été préparé et purifié au Laboratoire des Médiateurs chimiques. L'imprégnation des capsules a été réalisée à l'aide de solutions dans l'hexane (solvant seul pour les témoins), sans additifs (antioxydant, antiU.V.)

Les pièges ont été disposés à $1,60 \mathrm{~m}$ du sol (sauf cas indiqués) suivant une orientation approximativement parallèle au vent dominant et en respectant une distance minimum de $50 \mathrm{~m}$ entre 2 pièges. A chaque relevé quotidien, ils ont été soumis à une permutation circulaire (sauf cas indiqués).

Afin de faciliter les comparaisons, des répétitions (2 à 5) de formulations ont été faites dans plusieurs cas. La plupart des résultats de captures ont été analysés à l'aide de traitements statistiques (tests paramétriques ou non paramétriques).

\section{RÉSULTATS ET DISCUSSIONS}

A. Attractivité comparée des énynes $\mathbf{Z}(1)$ et $\mathbf{E}$ (2)-Site de Berganton, Cestas-Pierroton) 
Le dispositif utilisé pour cette étude comprenait 8 pièges répartis sur un parcours en $U$ (surface intérieure : environ $1,5 \mathrm{ha}$ ) et distants de $1,5-2 \mathrm{~m}$ des arbres les plus proches. Il a été installé du $1^{\text {er }}$ juillet au 12 août 1981 , dans un peuplement ouvert et en lignes de pins âgés de 9 ans (hauteur moyenne : $5 \mathrm{~m}$ ) avec un sous-bois de molinie, à une distance d'environ $30 \mathrm{~m}$ de la lisière ouest. Cette zone était fortement infestée par $T$. pityocampa en 1980.

Les résultats des captures (tabl. 1, fig. 3 et 4 ) indiquent un fort pouvoir attractif de l'ényne $Z(1)$. L'ényne isomère (2) l'est cependant également mais beaucoup moins, (différence significative au seuil de 5 p. 100) et il n'est pas exclu que cette attractivité soit due au faible taux d'isomère $Z$ contenu dans (2). L'ényne Z à 4 p. 100 d'isomère $E$ semble avoir une activité légèrement inférieure à celle qu'il présente lorsque son degré de pureté est supérieur (0,6 p. 100 E).

L'ényne $\mathrm{Z}$ ( $l$ ) qui a été identifié dans les extraits de glandes phéromonales de $T$. pityocampa est bien le constituant principal de la sécrétion. L'isomère $E$ (2) est soit faiblement attractif, soit inactif, soit faiblement inhibiteur. D'autres expériences seront nécessaires pour choisir plus précisément parmi ces hypothèses.

\section{B. Rôle de l'acétate de dodécanyle (3) ou DDA (parcelle M, Pierroton)}

Un ensemble de 8 pièges répartis sur un parcours circulaire a été disposé du 29 juin au 2 août 1981 , dans un peu-

\section{TABLEAU 1}

Influence de la géométrie de l'ényne ( $Z$ ou E) sur les captures de mâles de T. pityocampa (1.07 au 12.08.81)

Effect of $(Z$ or E) enyne geometry on male catches

\begin{tabular}{|c|c|}
\hline FORMULATIONS & $\begin{array}{l}\text { Captures totales } \\
\text { de mâles (1) }\end{array}$ \\
\hline $\begin{array}{r}-1.000 \mu \mathrm{g} \text { d'ényne } \mathrm{Z} I(0,6 \mathrm{p} .100 \mathrm{E}) \\
\text { piège } 1 \\
\text { piège } 2\end{array}$ & $\begin{array}{ll}127 \\
117\end{array}$ \\
\hline $\begin{array}{r}-1.000 \mu \mathrm{g} \text { d'ényne } \mathrm{Z} I(4 \mathrm{p} .100 \mathrm{E}) \\
\text { piège } 1 \\
\text { piège } 2\end{array}$ & $\begin{array}{ll}81 \\
90\end{array}$ \\
\hline $\begin{array}{r}-1.000 \mu \mathrm{g} \text { d'ényne E } 2(4 \mathrm{p} .100 \mathrm{Z}) \\
\text { piège } 1 \\
\text { piège } 2\end{array}$ & $\begin{array}{ll}26 & b \\
31 & \end{array}$ \\
\hline - Témoin & $0 \mathrm{c}$ \\
\hline
\end{tabular}

(1) Les captures affectées d'une même lettre ne sont pas significativement différentes au seuil de 5 p. 100 (test de Duncan)

plement clair de pins âgés de 30 ans (hauteur moyenne : $24 \mathrm{~m}$ ), avec un sous-bois de molinie, peu infesté par $T$. pityocampa en 1980. La distance des pièges aux arbres était au minimum de $2 \mathrm{~m}$. Les formulations, mises en comparaison $(1.000 \mu \mathrm{g}$ d'ényne $\mathrm{Z} 1(0,6 \mathrm{p} .100 \mathrm{E}) ; 1.000 \mu \mathrm{g}$ d'ényne $Z 1(0,6$ p. $100 \mathrm{E})+50 \mu \mathrm{g}$ de DDA 3$)$ sur cette

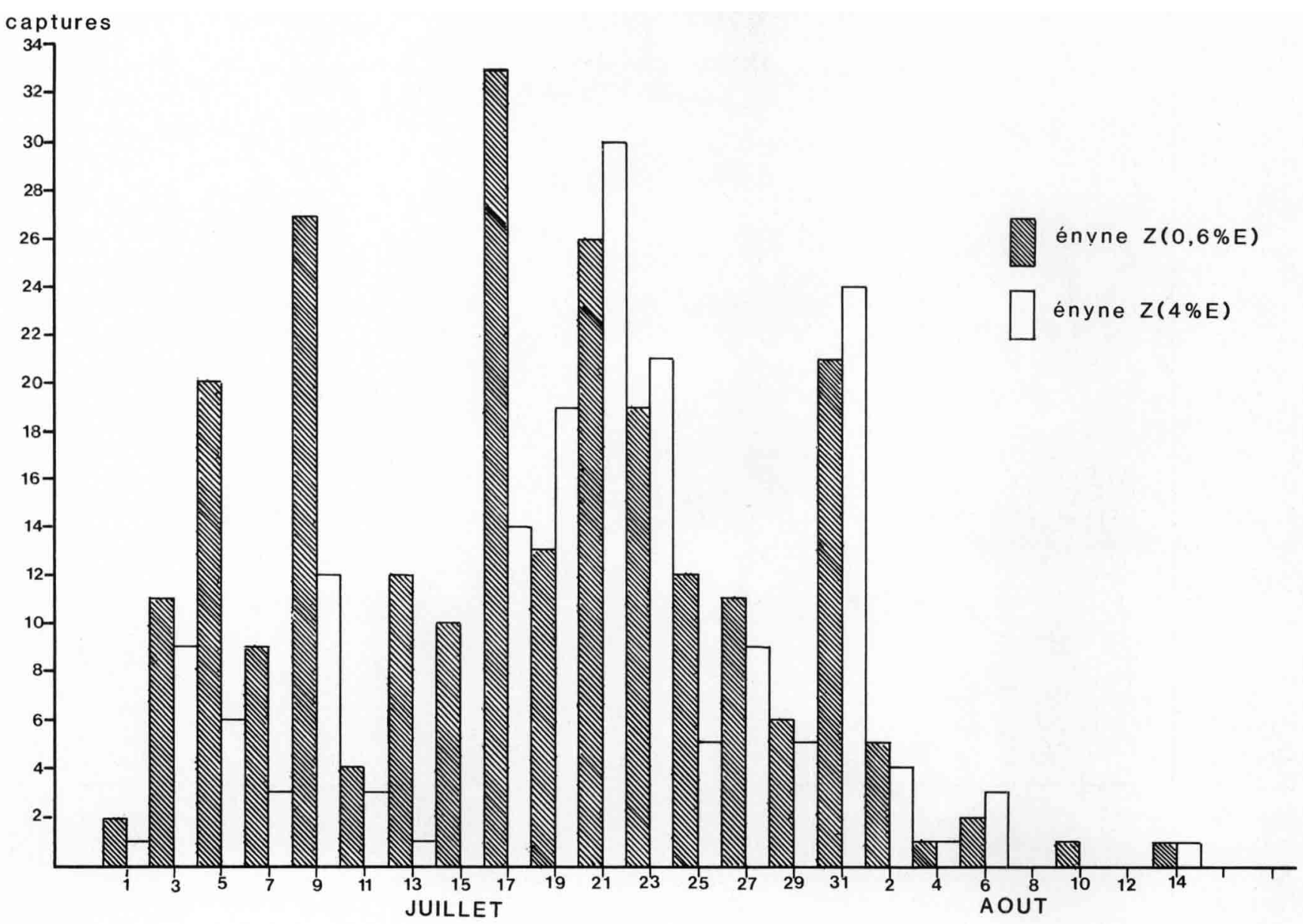

Figure 3

Influence de la géométrie de l'ényne (histogrammes).

Effect of enyne geometry (histrograms). 


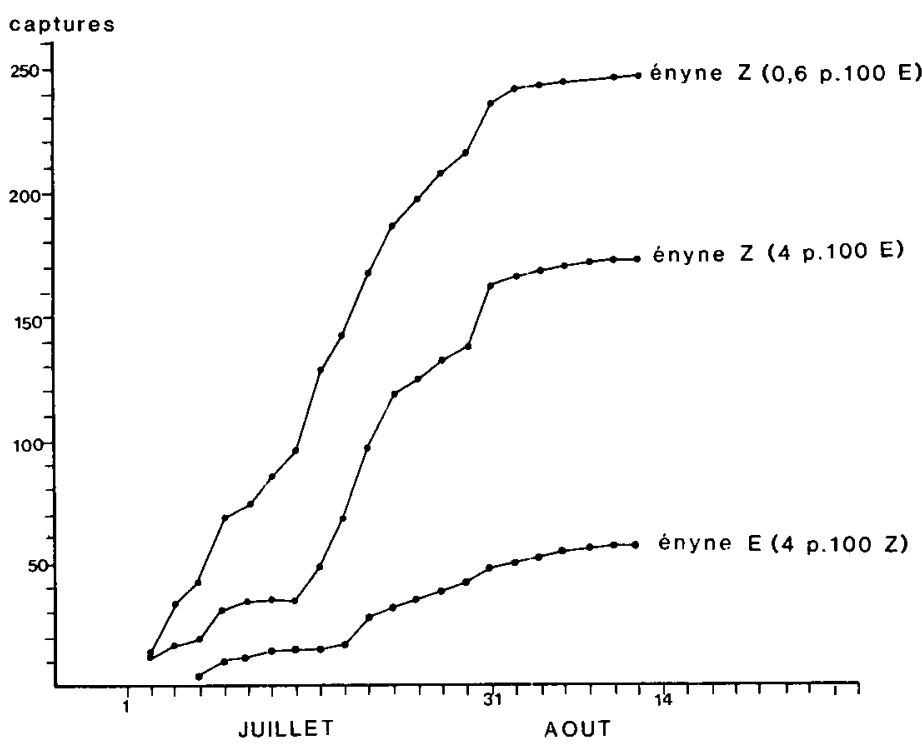

a)

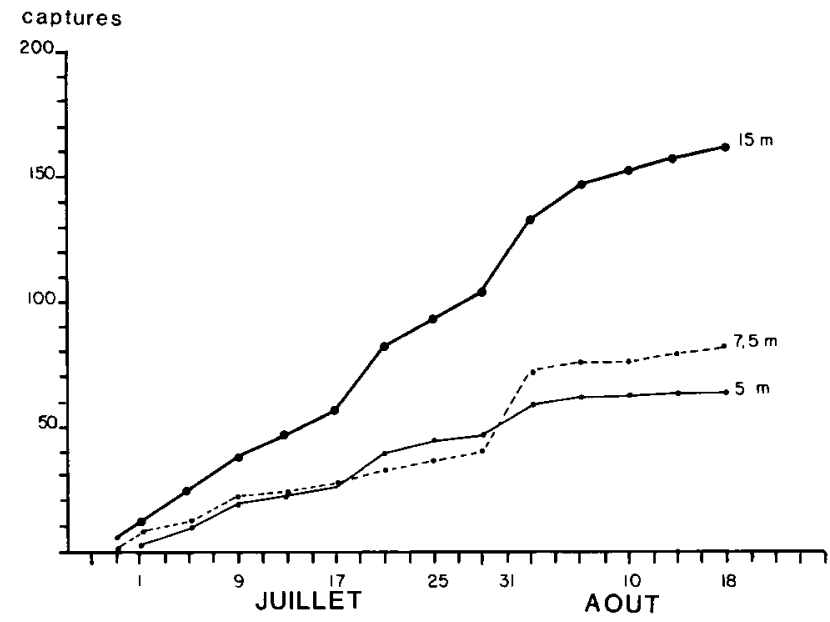

b)

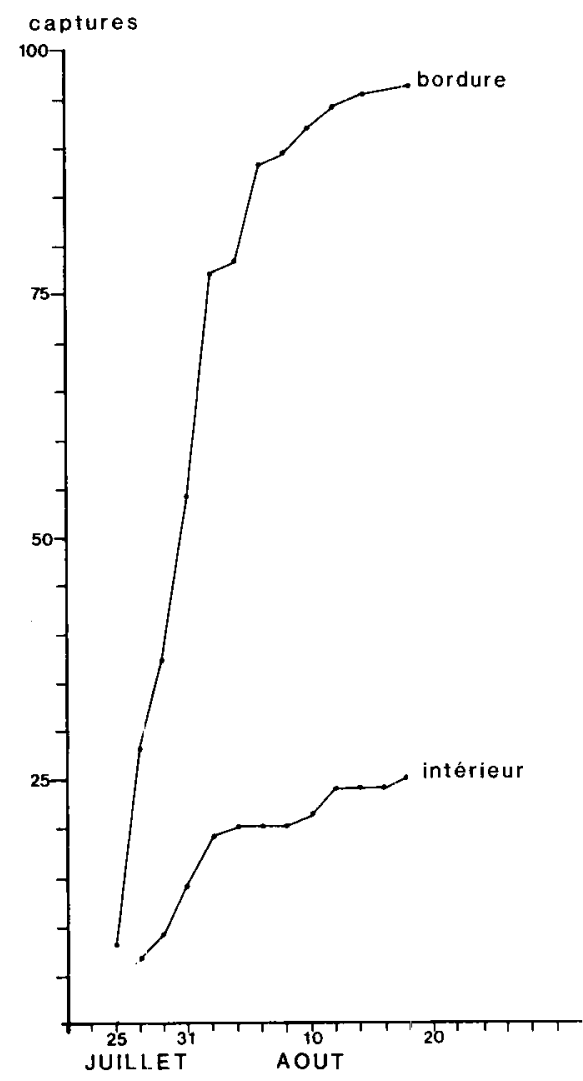

c)

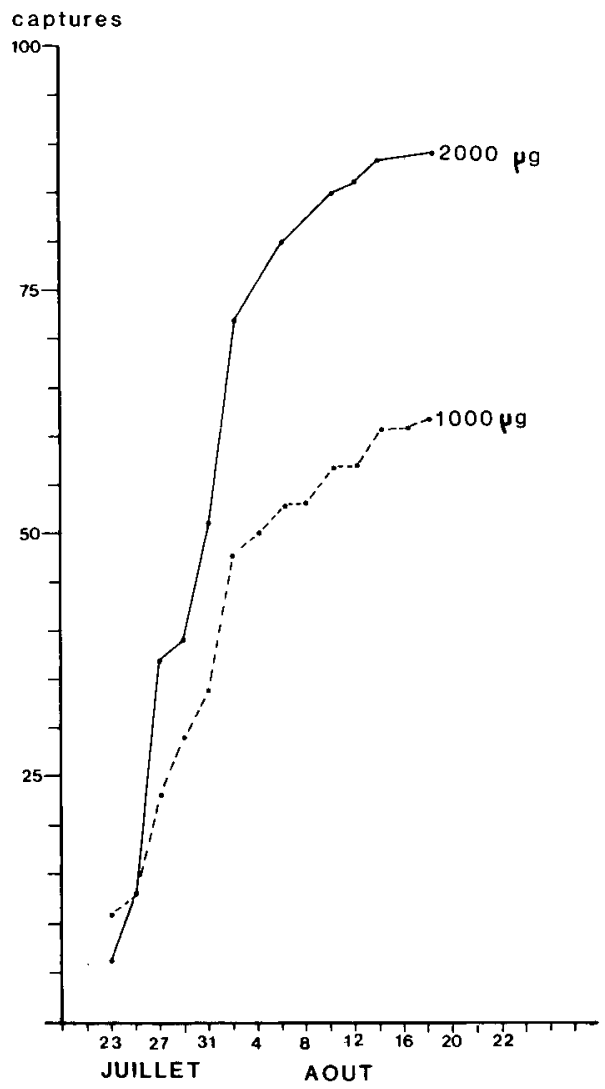

d) 
parcelle, étaient destinées à mettre en évidence le rôle éventuel du composé (3) dans la sécrétion phéromonale.

Les captures observées ont confirmé l'attractivité de l'ényne 1 , mais leur faible niveau général n'a pas permis de dégager de différences significatives. On a pu toutefois conclure à un effet non inhibiteur de l'acétate de dodécanyle.

\section{Influence de la dose (parcelle $\mathbf{T}$, Pierroton)}

Un dispositif en cercle de 8 pièges, destiné à comparer 2 doses de phéromone était installé le 21 juillet 1981 dans un peuplement clair de pins âgés de 25 ans (hauteur maximum : $20 \mathrm{~m}$ ), avec un sous-bois de molinie et de fougère. Les pièges, disposés à $2 \mathrm{~m}$ des arbres les plus proches, étaient munis de capsules contenant 1.000 ou $2.000 \mu \mathrm{g}$ d'ényne Z 1 (0.6 p. $100 \mathrm{E})$, de façon alternée le long du dispositif.

Le doublement de la dose de phéromone dans les capsules entraîne une augmentation sensible du niveau des captures (fig. 4). La différence n'est cependant pas significative au seuil de 5 p. 100 , du fait vraisemblablement de l'installation tardive du dispositif par rapport à la période de vol de l'insecte.

\section{Hauteurs de piégeage (parcelle T, Pierroton)}

Il a été montré, notamment pour Cydia pomonella L. (RIEDL et al., 1979 ; Mc NALLY \& BARNES, 1981) et Synanthedon pictipes Grote et Robinson (Mc LAUGHLIN et al., 1976), que la hauteur de piégeage pouvait avoir une influence importante sur le niveau des captures des mâles de l'espèce considérée. En ce qui concerne $T$. pityocampa, l'étude de ce facteur était de surcroît intéressante à aborder compte tenu du cycle biologique particulier de l'insecte, impliquant, dès l'émergence des adultes, le passage d'une phase souterraine à une phase aérienne.

3 pièges ont été disposés le 27 juin 1981, en lisière sur des pins de 34 ans (de 20 à $25 \mathrm{~m}$ de haut) à $1 \mathrm{~m}$ du tronc, à 3 hauteurs différentes : $15 \mathrm{~m} ; 7,5 \mathrm{~m} ; 5 \mathrm{~m}$. Les capsules étaient chargées, dans les 3 cas, à $1.000 \mu \mathrm{g}$ d'ényne $\mathrm{Z} 1$ $(0,6$ p. $100 \mathrm{E})$. Le dispositif a été maintenu jusqu'au 18 août.

La permutation circulaire des pièges n'étant intervenue qu'à partir du 29 juillet, les résultats (fig. 4) ont été analysés (tests non paramétriques) sur 2 périodes : avant et après le 29 juillet. Dans les 2 cas, on conclut que le nombre de mâles captur és à $15 \mathrm{~m}$ est supérieur (différence significative au seuil de 5 p. 100) au nombre de mâles capturés à $5 \mathrm{~m}$ (ou même à $7,5 \mathrm{~m}$ ). Il est évident que l'interprétation de ces résultats nécessiterait des études comportementales approfondies ; on peut cependant remarquer que le maximum de captures s'est effectué au niveau du houppier.

\section{E. Influence de la situation en lisière ou à l'intérieur du peu- plement (parcelle Q, Pierroton)}

Afin de mettre en évidence un « effet de bordure », un double dispositif a été mis en place (23 juillet - 18 août 1981) dans un peuplement fermé et en ligne de pins âgés de 15 ans (hauteur moyenne ; $12 \mathrm{~m}$ ), avec un sous-bois de molinie. Appâtés chacun à l'aide de $1.500 \mu \mathrm{g}$ d'ényne $Z l(0,6 \mathrm{p} .100$ E), 5' pièges étaient disposés en cercle, à $2 \mathrm{~m}$ des arbres les plus proches au sein du peuplement, tandis que 5 autres étaient placés en ligne, à $5 \mathrm{~m}$ des arbres de la lisière ouest (moyennement infestée en 1980). Les 2 systèmes de pièges, distants entre eux d'au moins $60 \mathrm{~m}$, ont subi séparément des permutations circulaires.

Le système de piégeage en bordure a capturé de façon significative (seuil de 1 p. 100, paramétrie) un plus grand nombre de mâles que le système situé à l'intérieur de la parcelle (fig. 4).

\section{F. Comparaison au piégeage lumineux (6 postes du réseau " piégeage lumineux » du Sud-Ouest de la France)}

Des pièges sexuels ( $1.000 \mu \mathrm{g}$ d'ényne $\mathrm{Z} 1$ à $0,6 \mathrm{p} .100 \mathrm{~d}$ 'isomère $E$ ) ont été installés sur 6 sites déjà pourvus d'un piège lumineux (lampe d'appel à vapeur de mercure de $160 \mathrm{~W}$; lampe de capture : 2 tubes actiniques de $6 \mathrm{~W}$ ). Sur chaque site, les emplacements des 2 types de pièges ont été choisis de telle sorte que des écrans de végétation ou des bâtiments réduisent au maximum les interactions éventuelles (distance entre les 2 pièges comprise entre 300 et $2.000 \mathrm{~m}$ ). Compte tenu de l'aspect exploratoire de cette campagne de piégeage, aucune permutation entre les pièges lumineux et sexuel d'un site n'a été effectuée.

Le tableau 2 indique les résultats comparés des captures pour des périodes communes. Les pièges des sites 5 et 22 ont fonctionné durant toute la période de vol. Dans les autres sites, seule la fin du vol a été enregistrée. Il est à remarquer également que les pièges lumineux utilisés ne capturent que des mâles de processionnaire du pin.

L'examen des courbes de piégeage (tabl. 2, fig. 5) permet de faire plusieurs constatations. Dans la plupart des sites, on note une courbe moins étendue avec le piège sexuel qu'avec le piège lumineux. En effet, la fin des captures au piège sexuel peut précéder de 1 à $9 \mathrm{j}$ celle des piégeages lumineux. Le phénomène est toutefois inversé sur le site 22 (près d'Angoulême). Mais, il faut alors remarquer que le " retard " du piège sexuel qui était de $8 \mathrm{j}$ au niveau $50 \mathrm{p}$. 100 n'était plus que de $2 \mathrm{j}$ au niveau $90 \mathrm{p}$. 100. On peut constater également que certaines «pointes de capture » (maxima) observées en piégeage lumineux sont absentes ou peu marquées dans les courbes de captures correspondantes du piégeage sexuel (site 5,30 juillet et jours suivants ; site 8,30 juillet et 4 août ; site 10,4 août ; site 14,29 juillet ; site 22, 29 juillet au 4 août). D'autres observationis seront néces-

\section{TABLEAU 2}

Captures totales et pourcentages cumulés de captures de mâles de T. pityocampa ( $L=$ piège lumineux; $S=$ piège sexuel; $\triangle j=$ ecart (jours) entre pièges lumineux et sexuel au niveau indiqué)

Total and cumulated per cent catches of $\mathrm{T}$. pityocampa males $L=$ light trap ; $S=$ sexual trap ; $\triangle j=$ difference (days) between light and sexual trap at a given level

\begin{tabular}{|c|c|c|c|c|c|c|c|c|c|}
\hline \multirow[t]{3}{*}{$\begin{array}{l}\text { Site } \\
\left(n^{\circ}\right)\end{array}$} & \multirow[t]{3}{*}{ Période } & \multirow{2}{*}{\multicolumn{2}{|c|}{$\begin{array}{c}\text { Captures } \\
\text { totales }\end{array}$}} & \multicolumn{6}{|c|}{ Niveaux (p. 100 cumulés) } \\
\hline & & & & \multicolumn{3}{|c|}{50 p. 100} & \multicolumn{3}{|c|}{90 p. 100} \\
\hline & & $\mathrm{L}$ & $\mathrm{S}$ & $\mathbf{L}$ & $S$ & $\Delta \mathrm{J}$ & $\mathbf{L}$ & $S$ & $\Delta \mathrm{J}$ \\
\hline 5 & $6 / 7-25 / 8$ & 965 & 108 & $29 / 7$ & $20 / 7$ & +9 & $8 / 8$ & $30 / 7$ & + \\
\hline 8 & $22 / 7-31 / 8$ & 347 & 15 & $28 / 7$ & $24 / 7$ & +4 & $29 / 7$ & $29 / 7$ & \\
\hline 10 & $22 / 7-22 / 8$ & 440 & 90 & $30 / 7$ & $29 / 7$ & +1 & $12 / 8$ & $11 / 8$ & + \\
\hline 14 & $17 / 7-25 / 8$ & 1197 & 58 & $29 / 7$ & $24 / 7$ & +5 & $4 / 8$ & $29 / 7$ & + \\
\hline 18 & $20 / 7-31 / 8$ & 688 & 5 & $28 / 7$ & $28 / 7$ & 0 & $30 / 7$ & $29 / 7$ & + \\
\hline 22 & $10 / 7-31 / 8$ & 1000 & 357 & $4 / 8$ & $12 / 8$ & -8 & $25 / 8$ & $27 / 8$ & - \\
\hline
\end{tabular}



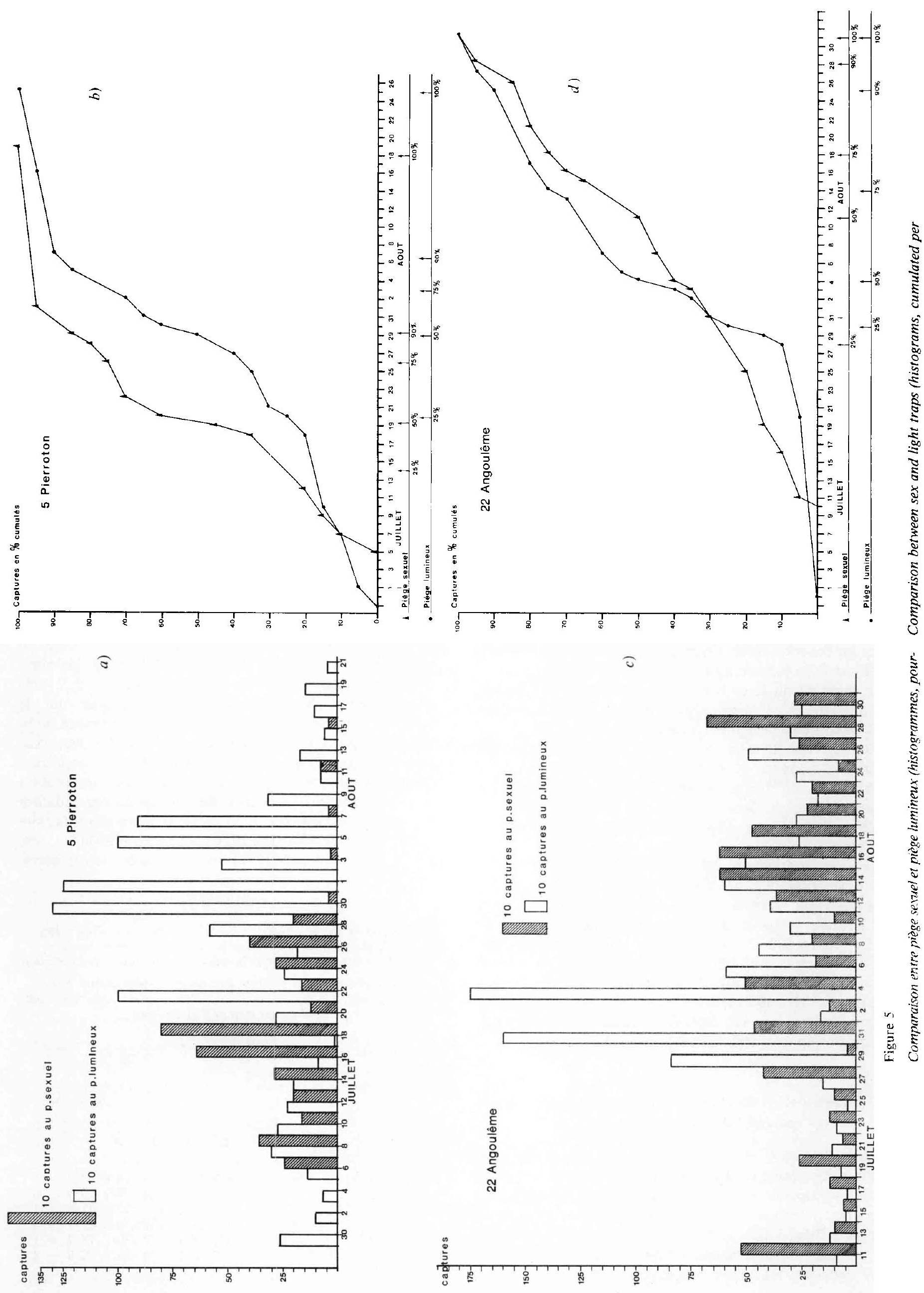
saires à la fois pour confirmer et interpréter ces phénomènes. Notons que si l'hypothèse d'une période de capture plus courte avec le piégeage sexuel devait se confirmer, ce résultat permettrait la prévision des dates de traitement avec une avance par rapport aux indications actuelles du piégeage lumineux et, par conséquent, de pouvoir intervenir dans de meilleures conditions parce qu'à plus longue échéance.

\section{CONCLUSION}

D'après nos différentes expérimentations, il résulte que l'acétoxy-1 hexadécen-13 (Z) yne-11 (I), découvert dans la sécrétion phéromonale de $T$. pityocampa, est bien attractif pour cette espèce; de plus, il s'est avéré spécifique dans tous les piégeages réalisés.

D'un point de vue pratique, la présence d'un faible pourcentage d'isomère $E(2)$ ne devrait pas influer fortement sur le niveau des captures et l'allure des courbes de piégeage (fig. 3). Par ailleurs l'optimisation du piégeage, notamment dans le cas de faible densité de population, de T. pityocampa, implique de prendre en compte les effets de dose, de hauteur et de bordure dont l'importance a été mise en évidence.
Enfin, les premières comparaisons entre piégeages sexuel et lumineux montrent que les 2 procédés ont donné en 1981 des informations assez comparables dans leur ensemble sur la période d'activité des adultes de $T$. pityocampa dans le massif forestier landais.

Sous réserve d'être encore confirmées et précisées lors de prochaines campagnes d'avertissement, ces premières données permettent déjà d'envisager de substituer, dans un proche avenir, le piège sexuel au piège lumineux pour assurer la surveillance de $T$. pityocampa et la protection de la pinède contre ce ravageur.

Reçu le 5 mars 1982. Accepté le $1^{\text {er }}$ février 1983.

\section{REMERCIEMENTS}

Nous remercions vivement le Dr A. GuErrero (Instituto de Quimica Bio-organica, Barcelone) de nous avoir fourni des énynes de synthèse, J.-P. CARles (I.N.R.A., Bordeaux) de ses conseils pour l'interprétation statistique des résultats ainsi que F. PAGEZy (stagiaire E.N.I.T.A.), Mme M. LeTtere (I.N.R.A., Laboratoire des Médiateurs chimiques) et J. DARNAUDERY pour leur collaboration à ce travail.

\section{RÉFÉRENCES BIBLIOGRAPHIQUES}

Camps F., Coll J., Canela R., Guerrero A., Riba M., 1981. Synthesis of the two isomers of the potential sex pheromone of Thaumetopoea pityocampa (Lep., Notodontidae) and related model compounds. Chem. Lett., 703-706.

Cuevas P., Montoya R., Belles X., Camps F., Coll J., Guerrero A., Riba M., 1981. Aplication del acetato de (Z)-13-hexadecen-11-inilo como agente de control potencial de la procesionaria del pino. Jornadas de Productos fitosanitarios, p. 77-83, 26-27 oct. 1981, Barcelona.

Demolin G., 1969. Comportement des adultes de Thaumetopoea pityocampa Schiff. Dispersion spatiale, importance écologique. Ann. Sci. for., 26, 81-102.

Guerrero A., Camps F., Coll J., Riba M., Einhorn J., Descoins Ch., Lallemand J.-Y., 1981. Identification of a potential sex pheromone of the processionary moth, Thaumetopoea pityocampa (Lep., Notodontidae). T. Letters, 22, 2013-2016.
Mc Laughlin J.R., Doolittle R.E., Gentry C.R., Mitchell E.R., Tumlinson J.H., 1976. Response to pheromone traps and disruption of pheromone communication in the lesser peachtree borer and the peachtree borer (Lep., Sessidae). J. chem. Ecol., 2, 73-81.

Michelot D., Guerrero A., Ratovelomanana V., 1982. An efficient stereoselective synthesis of (Z)-13-hexadecen-11-ynyl acctatc, a sex attractant of Thaumetopoea pityocampa Den, and Schiff. (Lep., Notodontidae). J. chem. Res. (S), 93.

Mc Nally P.S, Barnes M.M., 1981. Effects of codling moth pheromone trap placement, orientation and density on trap catches. Environ. Entomol., 10, 22-26.

Riedl H., Hoying S.A., Barnett w.W., Detar J.E., 1979. Relationship of within-tree placement of the pheromone trap to codling moth catches. Environ. Entomol., 8, 765-769.

Stockel J., 1976. Mise au point d'un piège sexuel « I.N.R.A. " pour insectes. In les phéromones sexuelles des Lépidoptères. Publ. I.N.R.A., Bordeaux, 12-14. 